\title{
APLICAÇÃO DE UM MODELO MOLECULAR DE BAIXO CUSTO NAS AULAS DE QUÍMICA, EM ESCOLAS ESTADUAIS DO MUNICÍPIO DE UBAJARA- CE
}

\author{
Ana Danielle Queiroz Melo, Alessandro Carneiro lima, Francisco Walison Lima Silva, \\ Ruslana Silva Pereira, Maria Lidiane Nascimento Sousa \\ ${ }^{1}$ Instituto Federal de Educação, Ciência e Tecnologia do Ceará - IFCE \\ <daniellequimica@yahoo.com.br>.<aclima6@hotmail.com>,<alysom_ubj@hotmail.com> \\ $<$ ruslanapereira@gmail.com>.<didy2017@gmail.com>
}

DOI: $10.21439 /$ conexoes.v12i3.1242

\begin{abstract}
Resumo. A Química é uma ciência essencialmente experimental e a ausência de um conhecimento prático dificulta a aproximação dos conhecimentos teóricos da realidade dos alunos, tornando assim o processo de ensino-aprendizagem difícil. Diante disso, o trabalho teve como proposta a construção de um modelo molecular a partir de materiais de baixo custo, preferencialmente recicláveis e de fácil acesso, a ser aplicado nas escolas de ensino médio público do município de Ubajara - CE, pertencentes à Coordenadoria Regional de Desenvolvimento da Educação 5 (CREDE) da Secretaria de Educação do Estado. Para confecção do modelo molecular foi utilizado papel higiênico, cola branca, tinta guache, pincel e palitos de cotonete. Ou seja, utilizando materiais de baixo custo, esse trabalho nos possibilitou refletir que é exequível usar meios alternativos para o ensino de Química facilitando desta forma a motivação e a compreensão dos alunos.
\end{abstract}

Palavras-chaves: Modelos. Ensino. Química.

\section{APPLICATION OF A LOW COST MODEL MOLECULAR IN CLASSES OF CHEMISTRY, IN STATE SCHOOLS OF THE CITY OF UBAJARA, IN CEARA}

\begin{abstract}
Chemistry is an essentially experimental science and the absence of practical knowledge makes it difficult to approximate the theoretical knowledge of the students' reality, making the teachinglearning process difficult. Thus, the paper was proposed to build a molecular model from inexpensive materials, preferably recyclable and easily accessible, to be applied in high schools public of the city of Ubajara, in Ceará, belonging to Regional Coordination to Development of Education 5 (CREDE) of the State Education Departament. To make the molecular model was used toilet paper, white glue, gouache paint, brush, swab sticks, pliers and wire. This is using low cost materials, this paper has enabled us to reflect that it is feasible use alternative means of chemistry teaching thus facilitating motivation and understanding of the students.
\end{abstract}

Keywords: Models. Teaching. Chemistry.

\section{INTRODUÇÃO}

Segundo Galiazzi e Gonçalves (2004) a experimentação motiva e desperta o diálogo na sala de aula e influência positivamente no aumento do interesse em aprender ciências, o que constrói um ambiente adequado para a relação professor aluno. Porém, para que isso ocorra, espera-se que o discente do curso de Quí- 
mica, na modalidade licenciatura, adquira habilidades para preparação de materiais didáticos alternativos, almeje auto aperfeiçoamento assíduo, consiga adaptarse as diferenças de cada aluno entre outros (BRASIL 2001). Além disso, espera-se que o mesmo seja capaz de compreender o processo de ensino aprendizagem relativo à prática escolar, utilizando contextualizações e métodos que favoreçam o desenvolvimento do conhecimento de forma crítica e participativa, contribuindo assim, de forma direta, para o desenvolvimento integral dos estudantes.

Para Maia, Silva e Wartha (2008, p. 1) "No Brasil, ensina-se ciência de forma abstrata e esse tipo de ensino não promove a alfabetização científica", fato este que deriva de um ensino superficial que por vezes apresenta deficiências metodológicas que costumam dificultar a aprendizagem dos alunos, dentre elas: o baixo incentivo a capacitação continuada do professor, o engessamento do conteúdo pelo cumprimento da grade curricular, a escassez de material didático complementar, a infraestrutura precária, a ausência de laboratórios nas dependências físicas das escolas, a superlotação de alunos por sala dentre outros.

Paralelamente o Ensino de Química com excelência no Brasil ainda é utópico, visto os grandes desafios apontados anteriormente e complementados por Wartha; Silva e Maia, ao abordarem que:

Tal realidade é um retrato da falta de compromisso dos governos municipal, estadual e federal para com a educação básica. Dessa forma, tornase difícil para o professor promover alguma transformação no processo de ensino-aprendizagem, considerando-se ainda o desestímulo em virtude da desvalorização social da carreira (baixo salário, falta de qualificação e atualização) (MAIA; SILVA; WARTHA, 2008, p. 1-2).

Diante desse cenário, na região da Ibiapaba, situada do norte do Estado do Ceará essa realidade não é diferente, com o agravamento da escassez de professores qualificados lotados nas escolas públicas estaduais, na maioria dos casos as salas são lideradas por professores leigos na disciplina, ou seja, não são dotados do diploma de licenciado em química, ou por professores ainda em formação acadêmica.

Além disso o nível de abstração dos alunos no entendimento de química é alto, o que contribui para o insucesso na compreensão da maioria dos conceitos discutidos na Educação Básica (CIPOLLA; FERRARI 2016).

O ensino de ciências e de química ocorre de forma majoritariamente teórica na sala de aula, o que gera um conhecimento abstrato e superficial dos assuntos abordados e, justifica em parte o elevado analfabetismo científico do País, entende-se que o primeiro contato com ciências e química deveriam desafiar os alunos a serem protagonistas do desenvolvimento de seus conhecimentos através da contextualizações, aplicações e soluções dos problemas pela simples observação de fenômenos naturais inseridos no cotidiano do aluno.

A proposta desse trabalho pode ser entendida como uma resposta a esse anseio e objetivou discutir essa problemática desde a formação inicial do Licenciando em química, futuro docente em sala que terá como maior desafio tornar o processo de ensino-aprendizagem de química mais acessível aos alunos, mais dinâmico, sólido e prazeroso.

Com isso, propõe-se concomitantemente ao ensino de ligações químicas na Educação Básica e até no Ensino Superior a confecção de modelos moleculares a fim de tornar as moléculas estudadas literalmente mais palpáveis. É sabido que modelos são particularmente significativos no ensino de conceitos químicos (CIPOLLA; FERRARI, 2016), além de fornecerem uma compreensão adicional da configuração espacial dos átomos o manuseio dos modelos oportuniza uma visão tridimensional (DECKER; ASP, 1955).

\section{METODOLOGIA}

O seguinte trabalho baseou-se em duas fases, na primeira foi realizada a produção de um modelo molecular de baixo custo, e na segunda houve a aplicação do mesmo em escolas da rede estadual de ensino para analisarmos a influência dos materiais didáticos alternativos no processo motivacional e participativo dos alunos nas aulas de química/ciências, já que "é de conhecimento dos professores de ciências o fato de a experimentação despertar um forte interesse entre alunos de diversos níveis de escolarização." (GIORDAN, 1999. p. 43).

Para construção do modelo molecular, conforme moléculas ilustradas na Figura 1 foi necessária à adoção de etapas, objetivando a padronização do modelo, a fim de otimizar à construção e padronizar a utilização dos materiais a disposição.

Na confecção do modelo foi utilizado papel higiênico, cola branca, tinta guache, pincel e palitos de cotonete, materiais de fácil acesso e baixo custo, sendo que o modelo proposto pode ainda desafiar os novos alunos a reproduzirem e o aperfeiçoarem, a cada novo período letivo. Todos os itens citados para a confecção dos modelos foram encontrados no comercio local de Ubajara.

$\mathrm{O}$ procedimento, detalhado em etapas, foi: 


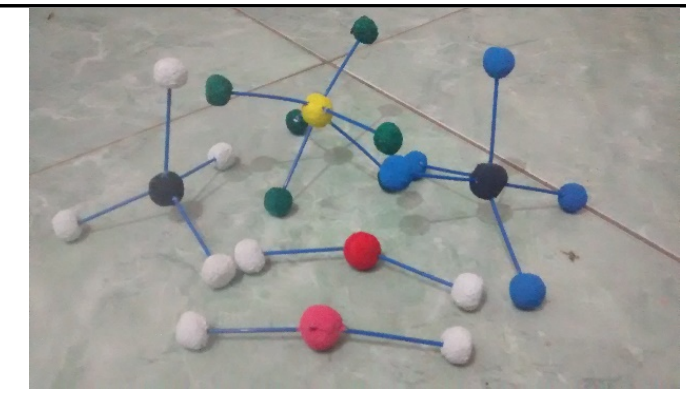

Figura 1: Exemplos de moléculas representadas pelo modelo molecular proposto.

Fonte: Próprio autor, 2016.

I - Umedeça o papel higiênico com água até o ponto que o mesmo adquira uma consistência pastosa, depois faça pequenas esferas (tamanho a critério), inserindo cola branca nas mesmas;

II - Após as esferas estarem prontas, aguardando somente a secagem, insira um palito de cotonete criando os orifícios que servirão como base para as ligações. Os orifícios devem estar na direção que respeite a geometria da molécula desejada. Espere secar;

III - Após a secagem, com o intuito de garantir a identidade dos elementos se fez necessário uma etapa adicional de pintura, ou seja, utilizando um pincel e tinta guache de diferentes cores as esferas (bolas de papel) devem ser coloridas e depois secas a temperatura ambiente;

IV - As ligações entre os elementos foram representadas com o auxílio de um palito. Quando necessário mais de um palito pode ser adicionado, no caso de ligações múltiplas. Outra forma para representar as ligações múltiplas, pode ser a utilização de palitos de cores diferentes;

$\mathrm{V}$ - Monte as moléculas desejadas, exemplos $\mathrm{CH}_{4}$ e $\mathrm{H}_{2} \mathrm{O}$, conforme ilustrado na Figura 2, sendo que a mesma metodologia pode ser adotada para a representação de quaisquer moléculas.

O modelo proposto apresenta as seguintes vantagens: ser um modelo simples, apresentar uma reprodução facilitada (visto que nas escolas praticamente não existem modelos, pois os modelos moleculares comerciais costumam ser caros e quando já foram adquiridos pelas instituições, não se apresentam em número suficiente), além de ser um modelo que permite alterações ou adaptações conforme a necessidade, por exemplo a Tabela de cores utilizada e demonstrada na Tabela 1 , pode ser adaptada e/ou ampliada.

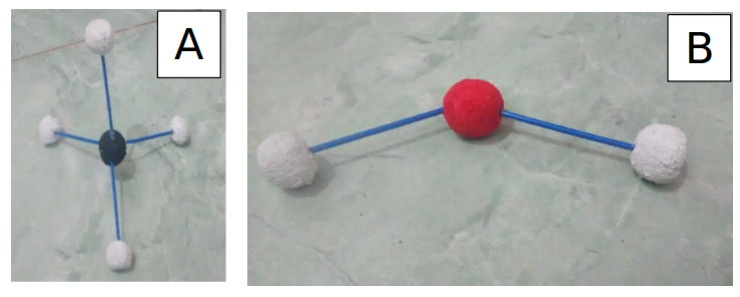

Figura 2: Moléculas de metano e da água, da esquerda para a direita, utilizando a metodologia de construção sugerida. Sendo A uma representação genérica de uma molécula de geometria tetraédrica e B a representação de uma molécula com geometria angular. Fonte: Próprio autor, 2016.

Tabela 1: Tabela de cores utilizada na confecção do modelo.

\begin{tabular}{ccc}
\hline $\begin{array}{c}\text { Símbolo } \\
\text { do } \\
\text { elemento }\end{array}$ & $\begin{array}{c}\text { Nome } \\
\text { do } \\
\text { elemento }\end{array}$ & $\begin{array}{c}\text { Cor } \\
\text { para } \\
\text { identificação }\end{array}$ \\
\hline Be & Berílio & Roxo \\
$\mathrm{B}$ & Boro & Rosa \\
$\mathrm{H}$ & Hidrogênio & Branco \\
$\mathrm{F}$ & Flúor & Verde \\
$\mathrm{C}$ & Carbono & Preto \\
$\mathrm{P}$ & Fósforo & Cinza \\
$\mathrm{S}$ & Enxofre & Amarelo \\
$\mathrm{Cl}$ & Cloro & Azul \\
$\mathrm{O}$ & Oxigênio & Vermelho \\
\hline \multicolumn{2}{c}{ Fonte: Próprio autor, 2016 }
\end{tabular}




\begin{abstract}
A tabela de cores confere uma identidade para cada elemento constituinte das moléculas, logo o modelo pode ser adaptado para ser originalmente orgânico ou inorgânico. Assim como a maleabilidade permite a representação de elementos com hibridização dos tipos $\mathrm{sp}, \mathrm{sp}^{2}$ e $\mathrm{sp}^{3}$.

Os alunos podem ser desafiados a reproduzirem os modelos e assim se tornarem protagonistas de aquisição de conhecimento e solidificação do conteúdo teórico por meio de atividades dinâmicas, colaborativas e interdisciplinares com o conteúdo geometria espacial da matemática, pois os ângulos entre as ligações estabelecidas dentre os átomos e pré-estabelecido pela teoria REPECV1 que prevê o tipo de geometria das moléculas
\end{abstract}

\section{RESUTADOS E DISCUSSÃO}

O modelo idealizado foi produzido pelos alunos do $3^{\circ}$ período do Curso de Química, na modalidade de licenciatura campus Ubajara do Instituto Federal de Ciências e Tecnologia (IFCE) foi aplicado na aula de ligações químicas e geometria molecular nas salas das turmas de $1^{\circ}$ ano A e B (conforme ilustrado na Figura 3) no ano letivo de 2016, na Escola Estadual de Ensino Médio Grijalva Costa, situada no município de Ubajara - Ceará.

Após a apresentação o modelo apresentou grande aceitação tanto por parte dos alunos quanto dos professores, pois além de possuir uma montagem facilitada, apresentam características essenciais: custo acessível, resistência mecânica e, principalmente, maleabilidade, pois como permite a saída das ligações, conforme a ilustração da Figura 4 o modelo permite uma aprendizagem dinâmica e prazerosa pois permite que os alunos ampliem alguns conceitos como as ligações químicas não são fixas, viabilizando o surgimento de rearranjos nas ligações ou ainda, o aparecimento de insaturações dentre outros.

Além disso, a saída das ligações possibilita montar e desmontar o modelo sempre que necessário, o que o torna um modelo prático que pode ser transportado e/ou armazenado com facilidade.

Ou seja, a possibilidade da visualização da disposição espacial dos átomos em ligações químicas estudadas na teoria pode ser considerada uma ferramenta didática poderosa, pois favorece uma maior interação entre os personagens no processo de ensino aprendizagem, aproxima os alunos dos conceitos teóricos, desperta o interesse pela disciplina dentre outras vantagens metodológicas.

\footnotetext{
${ }^{1}$ REPECV - Repulsão dos Pares de Elétrons da camada de Valência.
}

\section{CONCLUSÃO}

Ao final da construção e aplicação do modelo molecular proposto, avaliou-se o impacto gerado pela intervenção didática proposta com os discentes da escola estadual da cidade de Ubajara. Observou-se um maior interesse e motivação dos alunos na aprendizagem de ligações químicas. Segundo o Ministério da Educação em 2013, "a escola não consegue mais atrair jovens brasileiros", contrariando esse cenário foi verificado que os alunos interagiram positivamente durante a aplicação do modelo e após houve uma discussão, mediada pela equipe de aplicação e pelo professor conteudista da disciplina de química da Escola, em que os alunos fizeram analogias entre o conteúdo de ligações químicas (previamente discutido com os alunos) e os modelos que foram apresentados.

Estudos estatísticos do MEC entre os anos de 2002 e 2012, o Ensino Médio amarga a condição de ser a etapa da vida estudantil do alunado brasileiro com o maior índice de evasão. Sendo assim, de forma estratégica, a turma escolhida para a intervenção pedagógica proposta foram turmas do $1^{\circ}$ ano do Ensino Médio.

A proposta foi demostrar que ações simples como a construção de um modelo molecular foi suficiente para atrair o interesse dos alunos pela disciplina e assim tornar as aulas de química mais prazerosas e dinâmicas. A facilidade na construção do modelo molecular capacita tanto o professor, como os alunos na reprodução e ainda a construção do modelo demonstrou ser uma atividade pedagógica cooperativa a partir do envolvimento dos diferentes personagens envolvidos no processo de ensino aprendizagem.

A possibilidade de os alunos visualizarem as moléculas tridimensionalmente permite o entendimento e a previsibilidade da geometria correta para cada uma das moléculas, tornando a aprendizagem uma questão de assimilação e, não mais uma etapa de memorização embasada de conceitos abstratos.

\section{REFERÊNCIAS}

ADLER, A. D.; J., S. Precise atomic and molecular. Journal of Chemical Education, v. 41, n. 12, p. 656-658, 1964.

ANKER, R. M. Construction of molecular models. Journal of Chemical Education, v. 36, n. 3, p. 138, 1959.

ARDAC, D.; AKAYGUN, S. Effectiveness of multimedia-based instruction that emphasizes molecular representations on students' understanding of chemical change. Journal of research in science 

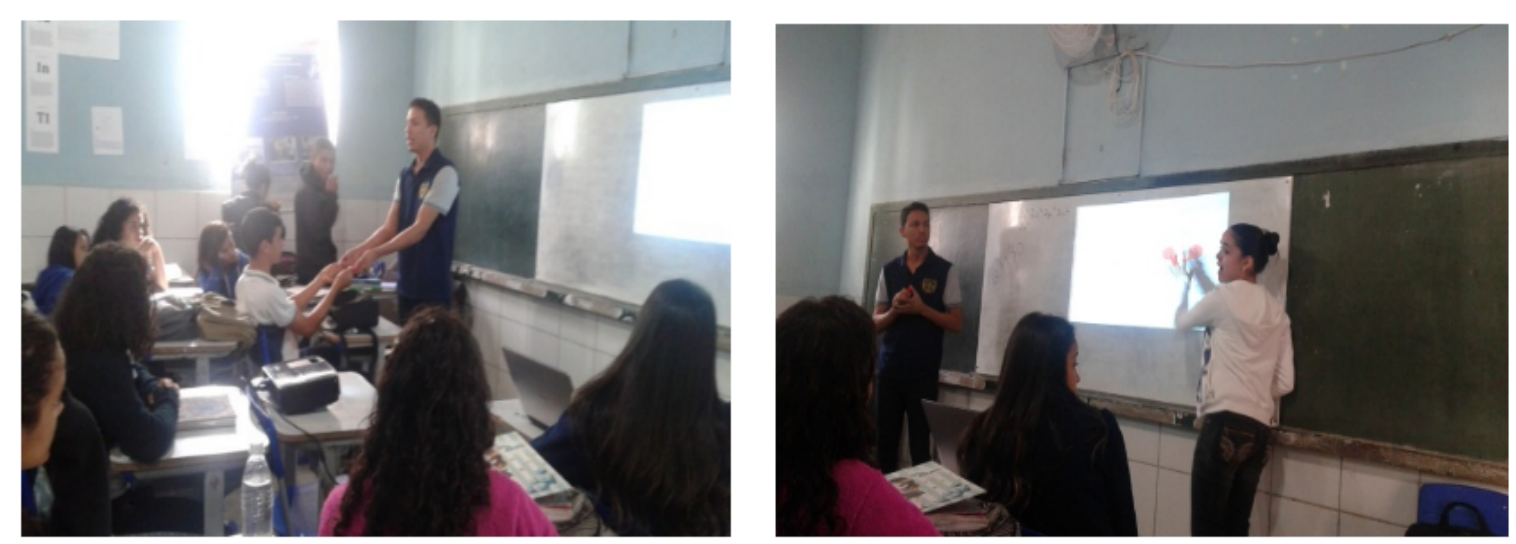

Figura 3: Fotos que demonstram o momento em que o modelo foi apresentado aos alunos. Fonte: Próprio autor, 2016.
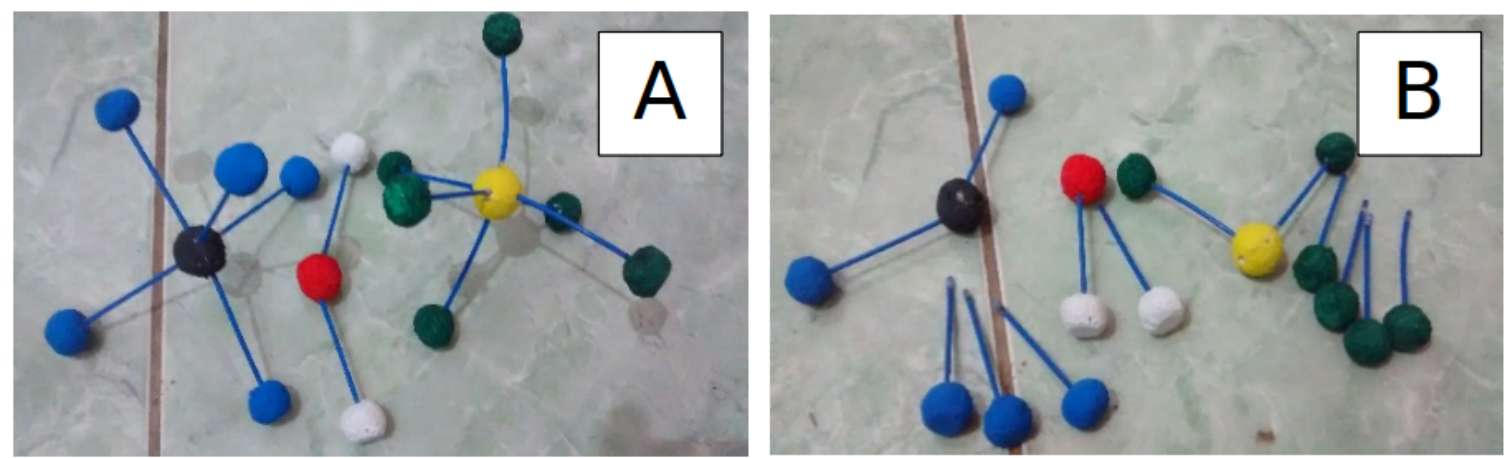

Figura 4: Saída das ligações. Sendo $\mathrm{A}=$ modelos montados e $\mathrm{B}=$ modelos desmontados. Fonte: Próprio autor, 2016. 
APLICAÇÃO DE UM MODELO MOLECULAR DE BAIXO CUSTO NAS AULAS DE QUÍMICA, EM ESCOLAS ESTADUAIS DO MUNICÍPIO DE UBAJARA- CE

teaching, Wiley Online Library, v. 41, n. 4, p. 317-337, GORDON, A. J. A survey of atomic and molecular 2004.

models. Journal of Chemical Education, v. 47, n. 1,

ATKINS, P.; JONES, L. Princípios de químicaquestionando a vida moderna e meio ambiente. 3 . ed. São Paulo: Bookman, 2007.

BRASIL. Lei n. 9.394. Lei de Diretrizes e Bases da Educação Nacional - LDB. Brasília: Congresso Nacional. 1996. Disponível em: <http: //www.planalto.gov.br/ccivil_03/leis/L9394.htm> Acesso em: 16 Fev 2017.

Parecer CNE/CES n. 1.303/2001. DIRETRIZES CURRICULARES PARA CURSOS DE QUÍMICA, BACHARELADO E LICENCIATURA PLENA. 2001. Disponível em: $<$ http://portal.mec.gov.br/cne/arquivos/pdf/CES1303. pdf> Acesso em: 16 Fev 2017.

Lei n. 13.005, de 25 de junho de 2014 . Aprova o Plano Nacional de Educação - PNE e dá outras providências. 2014. Disponível em: <http://www.planalto.gov.br/ccivil_03/ _Ato2011-2014/2014/Lei/L13005.htm.> Acesso em: 07 Abr 2015.

CARNEIRO, F. J. C.; RANGEL, J. H. G.; LIMA, J. M. R. Construção de modelos moleculares para o ensino de química utilizando a fibra de buriti. Acta Tecnológica, v. 6, n. 1, p. 17-26, 2011.

CHIPMAN, W. B. Molecular models. Journal of Chemical Education, v. 46, n. 2, p. 118, 1969.

CIPOLLA, L.; FERRARI, L. A. Big atoms for small children: Building atomic models from common materials to better visualize and conceptualize atomic structure. Journal of Chemical Education, ACS Publications, v. 93, n. 6, p. 1068-1072, 2016.

DECKER, B. F.; ASP, E. T. A jig for making atomic models. Journal of Chemical Education, v. 1, n. 1, p. 75-77, 1955.

GALIAZZI, M. d. C.; GONÇALVES, F. P. A natureza pedagógica das atividades experimentais: uma pesquisa no curso de licenciatura em química. Química Nova, SciELO Brasil, v. 27, n. 2, p. 326-331, 2004.

GIORDAN, M. O papel da experimentação no ensino de ciências. Química nova na escola, São Paulo, v. 10, n. 10, p. 43-49, 1999.

p. 30-32, 1970.

GUIMARÃES, C. C. Experimentação no ensino de química: caminhos e descaminhos rumo à aprendizagem significativa. Química nova na escola, v. 31, n. 3, p. 198-202, 2009.

HYDE, R. T.; SHAW, P. N.; JACKSON, D. E.; WOODS, K. Integration of molecular modelling algorithms with tutorial instruction. Journal of Chemical Education, American Chemical Society, v. 72, n. 8, p. 699, 1995.

LIMA, M.; LIMA-NETO, d. P. Construção de modelos para ilustração de estruturas moleculares em aulas de química. Química Nova, SciELO Brasil, v. 22, n. 6, p. 903-906, 1999.

MAIA, O.; SILVA, A. D. F. A.; WARTHA, E. J. Um retrato do ensino de química nas escolas de ensino médio de itabuna e ilhéus, ba. In: XIV Encontro Nacional de Ensino de Química (XIV ENEQ). [S.1.: s.n.], 2008. p. 1-11.

MARTINS, G. Atomic orbital molecular models Journal of Chemical Educatio, v. 41, n. 12, p. 658-661, 1964.

MASSI, L.; SANTOS, G. R. d.; FERREIRA, J. Q.; QUEIROZ, S. L. et al. Artigos científicos como recurso didático no ensino superior de química. Química Nova, Sociedade Brasileira de Química, v. 32, n. 2, p. 503-510, 2009.

MELO, M. R.; NETO, E. d. L. Dificuldades de ensino e aprendizagem dos modelos atômicos em química. Química Nova na Escola, v. 35, n. 2, p. 112-122, 2013.

ROQUE, N. F.; SILVA, J. L. d. P. B. A linguagem química e o ensino da química orgânica. Química Nova [online], v. 31, n. 4, p. 921-923, 2008.

SANDERSON, R. T. Atomic models in teaching chemistry. Journal of Chemical Education, v. 37, n. 6, p. 307-310, 1960.

SANTOS, H. F. O conceito da modelagem molecular. Cadernos temáticos de química nova na escola. São Paulo, v. 4, n. 1, p. 4-5, 2001.

STONE, A.; SIEGELMAN, I. An atomic and molecular orbital models kit. Journal of Chemical Education, v. 41, n. 7, p. 395, 1964. 